\title{
Does articulatory suppression eliminate the phonemic similarity effect in short-term recall?
}

\author{
JOHN T. E. RICHARDSON, DEBORAH E. GREAVES, \\ and MARGARET M. C. SMITH \\ Brunel University, Uxbridge, Middlesex UB8 $3 P H$, England
}

\begin{abstract}
Concurrent articulatory suppression does not appear to attenuate the effect of phonemic similarity upon short-term recall under conditions of auditory presentation. On theoretical grounds, the effect should be entirely abolished with visual presentation, but the available evidence is equivocal. The present study investigated the immediate serial recall of visually presented sequences of letters and words. Articulatory suppression reduced the overall performance and eliminated the phonemic similarity effect.
\end{abstract}

A well established finding in experiments on human short-term memory is that phonemic similarity among the stimulus items reduces the subjects' ability to remember the order in which the items were presented. This effect is usually attributed to the use of phonemic coding in short-term memory, and a primary source of such information is conventionally believed to be the subjects' vocalizations at the time of presentation. Consequently, if the subjects were required to produce vocalizations that were strictly irrelevant to the learning task, then their use of phonemic coding should be impaired, and this would be reflected in an attenuated effect of phonemic similarity in short-term recall. More specifically, under conditions of visual presentation, the subjects' vocalizations might be regarded as the only available source of phonemic information, and requiring the subjects to produce irrelevant vocalizations should completely eliminate the phonemic similarity effect.

These predictions relating to the effects of articulatory suppression were first considered by Murray (1967), who measured the immediate serial recall of visually presented sequences of letters when the subjects were required to vocalize the word "the" as each stimulus appeared. This reduced the subjects' overall performance and removed the effect of phonemic similarity upon recall. The phonemic similarity between the stimulus material and the irrelevant vocalizations was largely unimportant in determining the magnitude of the suppression effect. In a subsequent investigation, Murray (1968) considered the effect of articulatory suppression in immediate probed recall. Under conditions of visual presentation, vocalization of the word "the" produced impaired performance and reduced the phonemic

The authors are grateful to David Routh for his comments on an earlier draft of this paper and to Tammy Mindel for her assistance in analyzing the results of the experiment reported here. Requests for reprints should be addressed to John T. E. Richardson, Department of Psychology, Brunel University, Uxbridge, Middlesex UB8 3PH, England. similarity effect to a nonsignificant level. However, with auditory presentation, articulatory suppression had a much less pronounced effect, and it did not appear to reduce the magnitude of the phonemic similarity effect. These findings suggest that phonemic coding in shortterm memory is abolished with visual presentation if the subjects are prevented from vocalizing the material to be remembered, but that such coding may be established without vocalizing under conditions of auditory presentation.

An investigation by Levy (1971) also considered the immediate probed recall of visually presented sequences of letters. This study manipulated in an orthogonal fashion the availability of acoustic information (by introducing simultaneous auditory presentation) and the availability of articulatory information (by requiring the subjects to mouth silently either the stimulus items themselves or the "neutral" word "hi-ya"). Articulatory suppression led to poorer performance, but not when acoustic information was available; this suggested that both acoustic and articulatory features might be encoded in short-term memory. Although the main effect of phonemic similarity was highly significant, none of the interactions involving this factor was significant. So, while Levy did not actually present the mean scores from the relevant conditions of her investigation, one must conclude that it provided no support for the hypotheses stated above. A second experiment reported in her paper investigated the role of semantic similarity in the immediate probed recall of sequences of words. This confirmed the disjunctive relationship between the effects of auditory presentation and articulatory suppression, but neither factor showed a significant interaction with the effect of semantic similarity.

Peterson and Johnson (1971) considered the effects of phonemic similarity and of articulatory suppression in the immediate serial recall of sequences of five letters presented simultaneously on a memory drum for $2.2 \mathrm{sec}$. Relevant articulatory activity was suppressed by requiring the subjects to count aloud repeatedly from one to 
nine. This substantially reduced their overall performance and completely eliminated the effect of phonemic similarity upon recall. A second experiment presented the same sequences auditorily at a rate of 1 letter/sec. Counting aloud again reduced performance and showed a significant interaction with phonemic similarity, but the effect of the latter factor remained highly significant with articulatory suppression. This suggested that both auditory and articulatory cues could support short-term retention. Two further experiments investigated the effect of articulatory suppression using a form of the Brown-Peterson task. Counting aloud produced impaired performance with visual presentation, but not with auditory presentation, and only with short retention intervals.

The first two experiments reported by Peterson and Johnson (1971) appear to confirm the predictions stated earlier. However, a closer inspection of their results indicates that the use of visual presentation did not merely remove a source of phonemic information and thus reduce overall performance. Rather, although the phonemic similarity effect was attenuated with visual presentation whether or not the subjects were counting aloud, performance was actually improved in comparison with that obtained with auditory presentation, despite a shorter total presentation time $(2.2 \mathrm{sec}$ vs. $5 \mathrm{sec}$ ). This suggests that the use of simultaneous visual presentation in Peterson and Johnson's first experiment promoted a form of encoding that was distinct from, yet more efficient than, a phonemic representation of the stimulus material. An obvious candidate would be a visual or iconic code, and empirical findings relevant to this idea were obtained by Hiles (1974). Three experiments on the short-term recognition of visually presented letters showed that performance was impaired when a test letter was presented in a case different from that of the target letter, but only when vocalization of the stimulus items was suppressed. A fourth experiment showed that performance was impaired by phonemic similarity when vocalization was required, but by visual similarity when vocalization was suppressed. Hiles concluded that, if vocalization were prevented, subjects could encode visual material as a visual representation in short-term memory. This would explain the elimination of the phonemic similarity effect in Peterson and Johnson's (1971) study, and also the finding of Mowbray (1964) that concurrent shadowing impaired the retention of auditory material more than the retention of visual material.

Tell (1972) reported three experiments investigating the recall of visually presented consonant trigrams in the Brown-Peterson task. Articulation was suppressed by requiring the subjects to voice the words "three consonants" during the presentation interval of $.75 \mathrm{sec}$. Phonemic similarity and articulatory suppression affected performance with a short retention interval $(2.7 \mathrm{sec})$, but not with a long retention interval $(10.8 \mathrm{sec})$. Articulatory suppression also eliminated the phonemic similarity effect at the short retention interval. On the other hand, stimulus meaningfulness had a major effect upon recall with a long retention interval. Meaningfulness affected performance with a short retention interval only with phonemically similar material or when articulation was suppressed. Conversely, articulatory suppression had a greater effect upon the recall of material of low meaningfulness. Tell explained these findings in terms of a feedback-dependent echoic store and a semantically mediated long-term store: The influence of the latter component upon performance at short retention intervals was assumed to become apparent only when the contribution of the former was minimized, either by articulatory suppression or by high phonemic similarity.

Finally, Wilding and Mohindra (1980) investigated the effects of articulatory suppression and white noise upon the serial recall of visually presented sequences of letters. Articulation was suppressed by requiring the subjects to vocalize the word "the" repeatedly during the presentation of each sequence and throughout retention intervals of either $1.5 \mathrm{sec}$ or $8 \mathrm{sec}$. Articulatory suppression reduced the overall level of performance and removed both the effect of phonemic similarity and the effect of white noise upon recall. Its effect was greater at serial positions after the first in a sequence, at a faster rate of presentation, and with the longer retention interval.

One may now reconsider the predictions stated at the beginning of this paper. Of the three studies that have considered performance under conditions of auditory presentation, those of Levy (1971) and Murray (1968) failed to find an interaction between the effect of concurrent articulatory suppression and that of the phonemic similarity of the stimulus material. Peterson and Johnson (1971) did report a significant interaction between these two experimental manipulations, but their results indicate that this may have been caused by a floor effect in the suppression condition. The phonemic similarity effect was still highly significant in this condition, and it is probably safest to conclude that articulatory suppression does not attenuate the use of phonemic coding in short-term memory with auditory presentation. This suggests that acoustic and articulatory information are each individually sufficient for the use of such coding in short-term memory.

The situation is more complicated when one considers the findings obtained under conditions of visual presentation. Of the six relevant studies, those carried out by Murray $(1967,1968)$, by Peterson and Johnson (1971), by Tell (1972), and by Wilding and Mohindra (1980) appear to confirm the prediction that articulatory suppression eliminates the effect of phonemic similarity. However, the subjects in Peterson and Johnson's experiments appear to have utilized a form of visual encoding, and the investigation by Hiles (1974) confirmed that this is a possible consequent of articulatory suppression. More important, however, the experiment reported by Levy (1971) failed to show a significant interaction between the effects of articulatory suppression and of 
phonemic similarity, even with visual presentation. The following experiment attempted to clarify this issue in the immediate serial recall of sequences of letters and words.

\section{METHOD}

\section{Subjects}

Twenty-four undergraduates at Brunel University volunteered to participate in this experiment; 12 were male and 12 were female.

\section{Materials \\ The four sets of stimulus material were taken from Experi- ments 5 and 6 reported by Baddeley (1968). One set contained phonemically confusable letters $(b, c, d, p, t, v)$; another set contained phonemically distinct letters $(\mathrm{j}, \mathrm{k}, \mathrm{l}, \mathrm{r}, \mathrm{w}, \mathrm{y})$; a third set contained six phonemically confusable words (cad, can, cap, cat, mad, man); and the fourth set contained six phonemically distinct words matched for frequency with those in the previous set (bar, bun, day, few, rig, sup). Ten random orderings were derived of each of the four sets of stimuli. The two sets of 10 letter sequences were randomly allocated to two blocks, such that each block contained five phonemically confusable sequences and five phonemically distinct sequences, randomly ordered; the two sets of 10 word sequences were treated in a similar manner.}

\section{Procedure}

The stimulus material was initially displayed on the output scope of a PDP-12 computer; the items were presented in uppercase at a rate of 2 items/sec. Each trial was preceded by a sequence of warning signals and was followed by a recall signal. This was displayed for $20 \mathrm{sec}$ and was followed by a 10-sec blank interval before the commencement of the next trial. A videotape recording was made of this display and was shown to the subjects on 22-in. monochrome television monitors; the height of the resulting characters was approximately .75 in., and the monitors were viewed at a distance of approximately $5 \mathrm{ft}$.

The subjects were tested individually and were given standard serial recall instructions. Half of the subjects were presented with the sequences of letters, and half were presented with the sequences of words. Within each group, the relevant sequences were shown to all of the subjects in the same order. Independently of this, 12 subjects watched the first block of sequences in silence and pronounced the word "hi-ya" aloud repeatedly during the second block of sequences; the remaining 12 subjects vocalized during the first block and were silent during the second block. Three male and three female subjects contributed to each of the four conditions thus defined. The appropriate instructions were given immediately before each block of sequences; under articulatory suppression, the subjects were instructed to say "hi-ya" when each warning signal and each stimulus item appeared. At the end of each sequence, the subjects attempted to recall the items in silence by writing their responses on a prepared sheet, at the top of which were printed the 12 relevant stimuli (following the procedure of Baddeley, 1968).

\section{RESULTS}

In the control condition, when the subjects watched the material in silence, the mean percentage of items correctly recalled was $58.1 \%$ in the case of the phonemically confusable sequences and $74.9 \%$ in the case of the phonemically distinct sequences. The corresponding means for the experimental condition, in which the subjects repeatedly vocalized "hi-ya," were $43.2 \%$ and $44.3 \%$. Thus, the control condition demonstrated a clear effect of phonemic similarity, but the experimental condition did not. Conversely, there was a substantial effect of articulatory suppression upon performance, but this was reduced when the subjects had to remember phonemically confusable sequences of items.

These effects were investigated by means of an analysis of variance, carried out upon the number of items recalled in the correct serial position by each subject on each sequence. The basic design contained two random factors, subjects and sequences. The first random factor was nested under the fixed factors of sex (males vs. females) and order of conditions (silent first vs. vocalization first). The second random factor was nested under the fixed factors of blocks (first vs. second) and phonemic similarity. Both random factors were nested under the fixed factor of vocabulary (letters vs. words). The effect of articulatory suppression was identified as the interaction between the effect of blocks and the effect of order of conditions.

This analysis produced significant effects of phonemic similarity $[F(1,32)=11.70, p<.005]$ and of articulatory suppression $[\mathrm{F}(1,16)=52.03, \mathrm{p}<.001]$, and a significant interaction between these two effects $[F(1,32)=12.17, p<.005]$. Directional a posteriori tests (equivalent to one-tailed $t$ tests) showed that the effect of articulatory suppression was significant for both phonemically distinct sequences $[F(1,32)=71.02$, $\mathrm{p}<.001]$ and phonemically confusable sequences $[F(1,32)=16.80, p<.001]$; however, the effect of phonemic similarity was significant when the subjects were silent $[F(1,64)=23.69, p<.001]$, but not when they were required to vocalize "hi-ya" $[\mathrm{F}(1,64)=.10]$. There was a significant interaction between the effect of phonemic similarity and the effect of vocabulary $[F(1,32)=6.61, p<.02]$, indicating that the phonemic similarity effect was more pronounced with sequences of words than with sequences of letters. However, the effect of vocabulary did not qualify the effect of articulatory suppression $[F(1,16)=1.37, p>.25]$, nor was there a three-way interaction between these factors and the effect of phonemic similarity $[F<1]$. No other term in the analysis of variance was significant.

\section{DISCUSSION}

This experiment demonstrated substantial effects in immediate serial recall of both concurrent articulatory suppression and the phonemic similarity of the stimulus material. However, it also produced a clear and statistically reliable interaction between these two effects. Articulatory suppression produced poorer recall of both phonemically distinct and phonemically confusable sequences of items, but it also completely removed any sign of a difference in the performance obtained on the two sorts of stimulus material. This confirms the findings of Murray (1967, 1968), of Tell (1972), and of Wilding and Mohindra (1980) that, under conditions of visual presentation, articulatory suppression eliminates the effect of phonemic similarity. This is in contrast to the results typically obtained under conditions of auditory presentation, in which articulatory suppression does not seem to reduce the effect of phonemic similarity at all. Taken together, the empirical observations support the theoretical conclusion that the employment of phonemic coding 
in short-term memory requires the availability of either acoustic or articulatory information.

The idea that articulatory suppression abolishes the use of phonemic coding with visually presented material receives additional support from two further studies. First, Estes (1973) considered the recall of sequences of four letters after an interpolated delay occupied by an irrelevant activity. Each sequence contained two letters from a phonemically confusable subset and two letters from a nonconfusable subset, presented visually at a rate of $400 \mathrm{msec} /$ item. In the control condition, vocalization of the stimulus material was required; in the experimental condition, vocalization was suppressed by requiring the subjects to categorize each letter as occurring in the first or second half of the alphabet. Categorization produced impaired performance, but only at short retention intervals. Estes did not report separate recall scores for the confusable and nonconfusable stimulus materials. However, an analysis of the subjects' incorrect responses showed that phonemic confusion errors only occurred at short retention intervals and when vocalization of the stimulus material was required; there was no evidence of phonemic encoding of visually presented letters when vocalization was suppressed by the categorization task or when recall was delayed by more than $30 \mathrm{sec}$ of interpolated activity. These findings were subsequently replicated in a similar experiment by Healy (1975), in which vocalization was suppressed by requiring the subjects to say aloud the digits "one, two, three, four." Second, Baddeley, Thomson, and Buchanan (1975) considered the effect of word length upon performance in immediate serial recall. It was shown that performance was inversely related to word length across a variety of stimulus materials, which was taken as evidence for a phonemically based short-term store. Two experiments then considered the effects of articulatory suppression, produced by requiring the subjects to count aloud repeatedly from one to eight. With auditory presentation, there was a clear effect of word length, and this did not interact with the small decrement in performance caused by articulatory suppression. With visual presentation, however, articulatory suppression produced a substantial reduction in recall and completely eliminated the effect of word length. This implies that, under conditions of visual presentation, concurrent articulatory suppression removes the contribution to performance of the hypothesized phonemically based short-term store.

Finally, it should be pointed out that the effects of articulatory suppression upon short-term retention are rather different in tasks in which the subjects do not have to remember the temporal order in which the stimulus items were presented. For example, Healy (1975) found no effect of either phonemic similarity or delay upon the short-term recall of the spatial order in which a sequence of letters had been presented, and articulatory suppression had only a slight effect in that task. More important, Richardson and Baddeley (1975) showed that, in free recall, articulatory suppression produced a relatively small reduction in overall performance with both auditory and visual presentation and with both immediate and delayed testing. This pattern of results indicates that irrelevant vocalizations may exert a slight influence upon general processing capacity. Presumably, this is the locus of the effect of articulatory suppression upon immediate serial recall with auditory presentation, an effect that is rather tenuous and does not appear to be qualified by the phonemic similarity of the stimulus material (Levy, 1971; Murray, 1968; Peterson \& Johnson, 1971). Nevertheless, the disparity between the results obtained with free recall and those obtained with serial recall suggests that concurrent articulatory suppression exerts a substantial and relatively selective influence upon the retention of serial order information in a short-term phonemic store under conditions of visual presentation.

\section{REFERENCES}

Baddeley, A. D. How does acoustic similarity influence shortterm memory? Quarterly Journal of Experimental Psychology, 1968, 20, 249-264.

Baddeley, A. D., Thomson, N., \& Buchanan, M. Word length and the structure of short-term memory. Journal of Verbal Learning and Verbal Behavior, 1975, 14, 575-589.

Estes, W. K. Phonemic coding and rehearsal in short-term memory for letter strings. Journal of Verbal Learning and Verbal Behavior, 1973, 12, 360-372.

HEALY, A. F. Coding of temporal-spatial patterns in short-term memory. Journal of Verbal Learning and Verbal Behavior, 1975, 14, 481-495.

Hiles, D. R. Visual encoding in short-term memory (Doctoral dissertation, McGill University, 1973). Dissertation Abstracts International, 1974, 34, 6241 B.

LEvy, B. A. Role of articulation in auditory and visual shortterm memory. Journal of Verbal Learning and Verbal Behavior, 1971, 10, 123-132.

Mowbray, G. H. Perception and retention of verbal information presented during auditory shadowing. Journal of the Acoustical Society of America, 1964, 36, 1459-1464.

Murray, D. J. The role of speech responses in short-term memory. Canadian Journal of Psychology, 1967, 21, 263-276.

Murray, D. J. Articulation and acoustic confusability in shortterm memory. Journal of Experimental Psychology, 1968, 78, 679-684.

Peterson, L. R., \& Johnson, S. F. Some effects of minimizing articulation on short-term retention. Journal of Verbal Learning and Verbal Behavior, 1971, 10, 346-354.

Richardson, J. T. E., \& BadDeley, A. D. The effects of articulatory suppression in free recall. Journal of Verbal Learning and Verbal Behavior, 1975, 14, 623-629.

Tell, P. M. The role of certain acoustic and semantic factors at short and long retention intervals. Journal of Verbal Learning and Verbal Behavior, 1972, 11, 455-464.

Wilding, J., \& Mohindra, N. Effects of subvocal suppression, articulating aloud, and noise on sequence recall. British Journal of Psychology, 1980, 71, 247-261.

(Received for publication September 3, 1980.) 